\title{
Content based Trademark Retrieval by Integrating Shape with Colour and Texture Information
}

\author{
Akriti Nigam \\ Indian Institute of information \\ Technology, Allahabad
}

\author{
Arpit Kumar Garg \\ Indian Institute of information \\ Technology, Allahabad
}

\author{
R.C.Tripathi \\ Indian Institute of information \\ Technology, Allahabad
}

\begin{abstract}
Trademark is one of the most disputed items in Intellectual Property Rights. To grant a trademark, litigation is carried out to investigate if the new trademark applied for matches substantially with an already issued trademark. Considering the fact that the trademark database is very huge and keeps on increasing constantly, the manual search is definitely not a correct solution and an Information and Communication Technology (ICT) based system is required to automate this process.

In a content based retrieval system, shape is easily the most important feature for the trademark image. Using shape feature various systems have been developed in the past. Here, a new approach is proposed that integrates shape with color and texture information that improves the results significantly in terms of Precision and Recall. A flexible combination of features is used for improving performance and clustering is applied to the database to enhance retrieval efficiency.
\end{abstract}

\section{General Terms}

Trademark, Content Based Image Retrieval.

\section{Keywords}

Trademark Retrieval, color histogram, gray level co occurrence matrix, wavelet transform, Zernike moment, clustering.

\section{INTRODUCTION \\ 1.1 Background}

A trademark is a distinct sign used as an intellectual property by any individual, business organization or any other judicial entity. It can be an image, a logo, symbol, design, word, phrase or a combination of these. Trademark is basically used to distinguish one business organization from other and thereby distinguish their products or services from others as well [1].

Two trademark images can be conflicting not only if they are geometrically similar in a side by side comparison but also if they are visually similar in appearance. A trademark image can be suspected to cause infringement if it is similar to an already registered trademark image sufficient enough to arouse confusion. Similarity can be judged in numerous ways but the intention of a trademark retrieval system is to prevent trademark infringement.

Content Based Image Retrieval (CBIR) techniques make use of the visual information embedded in an image. This has been used to look for images in the trademark database which are confusingly similar to the query image requested by user. Using this visual content, also called features, a lot of human effort can be saved.

\subsection{Content Based Retrieval}

Trademark image retrieval techniques can be broadly classified as Description based trademark image retrieval and Content based trademark image retrieval.

In description based retrieval, the query is generated in the form of text describing the query image. The database images of all the classes along with their subclasses have annotations describing the image. In this case, the trademark image retrieval problem shrinks to the classical text search.

In content based retrieval [2] the query is in the form of image and its features are used as the content describing it. These features are calculated for the query image as well as for all the images in the database using feature extraction methods. After this, feature matching is done to find out and retrieve the similar images as shown in figure $\mathbf{1}$.

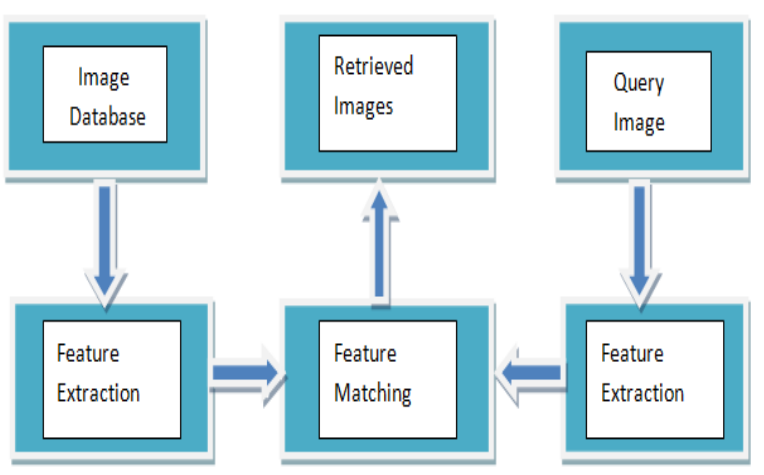

Figure 1 Block diagram of Traditional CBIR System

In a country like India, before year 1999, any trademark as per law was limited only to consider the graphical black and white images. However since the new trademark ser came in 1999, color trademark images are being widely used which are more attractive and convey more information and therefore a method that integrates the most relevant shape feature, with the color and texture information can prove to be a good solution. In our paper we here implement such a method.

\section{RELATED WORK}

A lot of work has been done in the field of trademark retrieval systems. The three most outstanding trademark retrieval systems are TRADEMARK [3], STAR [4] and ARTISAN [5].

Katos' TRADEMARK [3] system analyzes the image content automatically by making use of Graphical features (GF vector). Lam et al. developed STAR [4] system that makes use of mainstream image retrieval techniques that focus on both shape 
and spatial layout of image to calculate the similarity between images. These methods include Fourier descriptors, moment invariants and gray level projection.

ARTISAN [5] developed by Eakins, focuses on device-only marks which contain geometric designs which are abstract in nature and uses methods based on Gestalt psychology for similarity matching.

Apart from these three systems,QBIC [6] is a famous system developed by IBM, Almaden Research Center. It is in all probability the most efficient of all image content retrieval systems and can be used for both dynamic and static retrieval of images. It makes use of various visual properties like color, shape and texture.

S.Nandgopalan et al.[7] focused on color histogram, texture and image density features and used Euclidean distance method for similarity comparison between query image and database images.

Kondekar et al.[8] Presented results of efficiency of using color histogram, Fourier descriptors and moment invariants for color, shape and texture features respectively.

Rajshree S. Dubey et al.[9] proposed a system that used a combination of four feature extraction techniques namely color histogram, color moment, edge histogram and texture.

Before proceeding with this paper work an analysis of the survey done on various CBIR systems by Ranjeet Kumar et al. [10] has been made to get a better insight into the work already done in this field.

\section{PROPOSED METHOD}

In this paper we introduce a weighted approach that uses color, texture and shape as the basic features

\section{Feature Extraction Techniques \\ 3.1 COLOR}

\subsubsection{Color Space}

A color space defines a coordinate system and the subspace within the system. Each color is then represented as a point within that subspace. Some color spaces like RGB (red, green, blue), Lab (CIE L*a*b*, CIELAB), HSV (Hue, Saturation and Brightness), CMY (Cyan, Magenta,yellow) are used frequently. The color space used in computing the color histogram in the proposed method is HSV because HSV to RGB and reverse transformations are simpler and HSV color space is perceptually uniform.

\subsubsection{Color Histogram}

Color histogram [11] is a type of bar graph where on $\mathrm{X}$-axis we have a color and the frequency of occurrence of that particular color in the image is represented on Y-axis.

The steps followed to find the similarity between two images using color histogram are:

a) Query image and database images are read and converted to HSV color map.

b) Color histogram is computed for query and database images for all three components of HSV color space i.e. hue saturation and value.

c) Then distance between histograms is computed using Euclidean distance metric approach

This method of color histogram is invariant to image rotation.

\subsection{TEXTURE}

Texture [12] is an inherent property of an image that describes the homogenous pattern in the image. Texture is an important characteristic that specifies the coarseness, smoothness, uniformity etc. of objects in an image.

Much work has been done on analyzing textures but still it remains an interesting and difficult topic in image processing. The two common methods for describing textures are Statistical and Transform based methods.

\subsubsection{Statistical method}

This includes computing the local features at each point in the image using the gray level value at that point. Certain statistics is then derived from these local features. A large class of textures can be modeled by using Gray Level Co-occurrence matrix which is an effective statistical method.

\subsubsection{Gray Level Co-occurrence matrix}

Given an image $Q(i, j)$, let $p(i, j)$ be position operator. A matrix $A$ which is $N^{*} N$ in size is computed whose element $A(i, j)$ is the number of times that point with gray level (intensity) $g(i)$ occurs at a place specified by the relationship operator, $\mathrm{p}$, relative to points with gray level $g(j)$.

Suppose $\mathrm{P}$ be a $\mathrm{N}^{*} \mathrm{~N}$ matrix of size as of $\mathrm{A}$ and it is produced by dividing $\mathrm{A}$ with total number of point pairs that fulfill position operator $\mathrm{p} . \mathrm{P}(\mathrm{i}, \mathrm{j})$ is a joint probability that a pair of points fulfilling position operator $p$ will have values $g(i), g(j)$. $P$ is called the gray level co-occurrence matrix $[7,9]$ characterized by $\mathrm{p}$. The relationship operator $\mathrm{R}$ is characterized by angle $\theta$ and distance $\mathrm{d}$. 


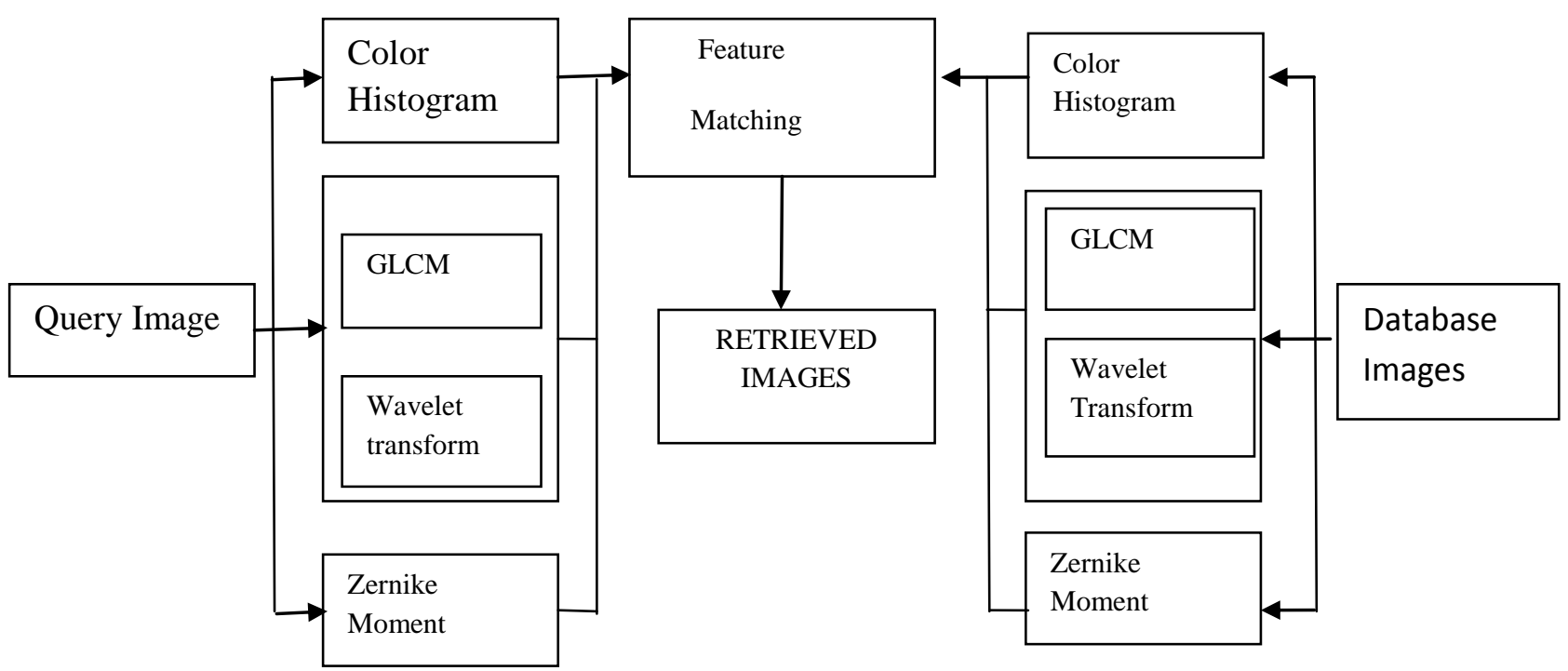

Figure 2 Flowchart of the proposed system

Using P following texture descriptors is computed:

Energy $=\sum_{i, j} P_{i, j}^{2}$

Inverse difference moment $=\sum_{\substack{i \neq j \\ i \neq j}} \frac{P_{i, j}^{2}}{\bmod (i-j)}$

Maximum probability $=\max \left(P_{i, j}\right)$

Contrast $=\sum_{i, j} \bmod (i-j)\left(P_{i, j}^{2}\right)$

Correlation $=\sum_{i, j} \frac{(i-\mu)(j-\mu) P_{i, j}}{\sigma}$

These statistic values are computed for the query image and the database images. The values are close for two similar images.

\subsubsection{Transform based methods}

In this method, the texture feature is computed by analyzing the frequency content of the image. Some methods use Fourier transform to compute the global frequency content of the image which work at a single scale, like the statistical methods. However there may be some texture patterns at different degree of details. To analyze these patterns multi resolution analysis is done. For this multi resolution analysis tree structured wavelet transform is used.

\subsubsection{Tree Structured Wavelet Transform}

Tree Structured wavelet transform [13] decomposes sub signals in the low frequency channels. This method is highly efficient because of the innate property of images because of which the essential information of images exists in lower sub bands. The wavelet function used is Daubechies.

Following steps are followed to find similarity between images based on texture:

After completion of distance computation for all the database images the similarity can be analyzed.

1. The texture image is decomposed into four sub regions in low-low, high-low, low-high and highhigh sub bands.

2. The energy level of each of the four sub bands is computed at the same scale using

$$
\mathrm{E}=\frac{1}{M N} \sum_{i=1}^{M} \sum_{j=1}^{N} \bmod (X(i, j))
$$

Where $M, N$ are dimensions of image and $X(i, j)$ is the intensity value of pixel at location $(i, j)$.

3. Steps 1 and 2 are repeated five times to reach fifth level of decomposition for the low low sub band image.

4. Obtain the energies of first $\mathrm{K}$ dominant channels for the image. 
5. Calculate Euclidean distance between two sets of energies of query and database image.

$$
\sum_{k=1}^{K}\left(X_{k}-Y_{k}\right)^{2}
$$

After completion of distance computation for all the database images the similarity can be analyzed.

\subsection{SHAPE}

Shape feature of an image can be calculated either by using its boundary or its region. Boundary based methods are not so much useful because they are based on single contour so they do not describe complex shapes appropriately.

Therefore, in this paper, region based descriptors such as moments are used because they rely on the pixels consisting region of a shape as well as contour pixels which make them more reliable for shapes having complex boundaries .Low order moments describe the global shape of an image while high order moments describe the shape in detail.

In the proposed method, Zernike moments [14] are used to compute the shape feature of an image.

\subsubsection{Zernike Moments:}

Zernike moments [14] are defined as complex number moment over a unit circle which takes a set or group of polynomials that are complex and orthogonal in nature.

For digital images, if $P_{x y}$ is current pixel then Zernike Moment [16] is calculated using following formula:

$A_{m n}=\frac{m+1}{\pi} \sum_{x} \sum_{y} P_{x y}\left[V_{m n}(\mathrm{x}, \mathrm{y})\right]^{*}$

where $\mathrm{m}=0,1,2 \ldots \infty$ and $\mathrm{n}$ depicts angular dependence

$V_{m n}(r, \theta)=R_{m n}(r) e^{j n \theta}$ are Zernike polynomials in polar Coordinates, with some conditions

$x^{2}+y^{2}=1, \mathrm{~m} \geq|n|,(\mathrm{m}-|\mathrm{n}|)$ is even and $A_{m n}^{*}=A_{m,-n}$

$R_{m n}(b)$ is orthogonal radial polynomial defined as

$R_{m n}(b)=\sum_{s 1=0}^{\frac{m-|n|}{2}}-1^{s 1} \frac{(m-s 1) !}{s 1 !\left(\frac{m+|n|}{2}-s 1\right) !\left(\frac{m-|n|}{2}-s 1\right) !} b^{m-2 s 1}$

$z 1=\left\|A_{m n}\right\|$ gives the magnitude of Zernike moment.

Since the basis of Zernike moments is orthogonal, they have some advantages over regular moments e.g. invariance in rotation, robustness against noise. Shapes with minor differences are identified in high order moments and information redundancy is also minimized. Also, when image description is done using moments, Out of Zernike Moments and other geometric moments, Zernike has better retrieval efficiency.

For calculation of Zernike moments of an image, what is done first, an image or interested region of an image has taken or identified then we map that region to the origin of unit circle in polar coordinate system
In calculation, the pixels internal to unit circle are considered. Rests of the pixels are excluded. Rotation invariance is achieved due to this mapping.

Before Calculating Zernike Moments, image is normalized using Cartesian Moments which will help in achieving scale invariance in scale and translation.

By using Euclidean distance, the Zernike moments values obtained for query image and for database images are compared to get the similar images based on shape.

\section{EXPERIMENTAL RESULTS}

\subsection{Clustering}

Here we suggest making small clusters of the trademark database images which aids in improving system efficiency.

Initially any number of clusters is taken arbitrarily, which for computational ease we have kept to 5. Cluster representatives are initialized and distance of each trademark image to these representatives is calculated and consequently the images are assigned to that cluster which has minimum distance and this process repeats by calculating new cluster representatives and this will continue until all the images find their correct representative.

After this process, when user presents the query image our system first finds which cluster the query image belongs to and then calculates total number of pertinent images to that query image in the trademark dataset .If suppose the query image should fall in the Pth cluster then all trademark images in the Pth cluster are relevant images in database, (let $N_{P}$ be that number) which helps in calculating the recall of a system i.e.

$$
\text { Recall }=\frac{N_{R}}{N_{P}}, N_{R} \rightarrow \text { no. of relevant images retrieved. }
$$

For Precision $=\frac{N_{R}}{N_{T}}, N_{T}$ is no. of retrieved images which is fixed to 10 . Table 1 below shows the recall and precision values obtained by taking each feature alone at a time.

\begin{tabular}{|l|l|l|l|}
\hline & Color & Texture & Shape \\
\hline Precision & 43.4 & 24.6 & 64.3 \\
\hline Recall & 54.6 & 32.8 & 67.5 \\
\hline
\end{tabular}

Table 1 Recall and precision in percentage by taking each feature alone at a time.

\subsection{Combining the features}

Getting the retrieval result using only single feature may produce inefficient result. It may either retrieve images not similar to query image or may fail to retrieve images similar to query image. Hence to produce efficient results, we used combination of several features instead of using single feature. 
As each feature has its different value so they are combined using variable weights using the following formula:

$\mathrm{S}=\frac{W_{C} \cdot D_{C}+W_{T} \cdot D_{T}+W_{S} \cdot D_{S}}{W_{C}+W_{T}+W_{S}}$

$W_{C}, W_{T} \& W_{S}$ are the contribution factors assigned to distance values $D_{C}$ for color $D_{T}$ for texture and $D_{S}$ for shape respectively.

The conclusion we derive from table 1 is that the retrieval precision based on shape is highest. After shape, color has a better retrieval precision result. Using this result we assign higher weight to shape feature, next highest to color and lowest to texture. The recall and precision obtained using this weighted combination is shown below

\begin{tabular}{|l|c|}
\hline & Proposed method \\
\hline Precision & 67.3 \\
\hline Recall & 75.3 \\
\hline
\end{tabular}

Table 2 Recall and precision values of the suggested system improving computational efficiency

\subsection{Database}

The database consists of India Trademarks mainly from class 16 and 38 .
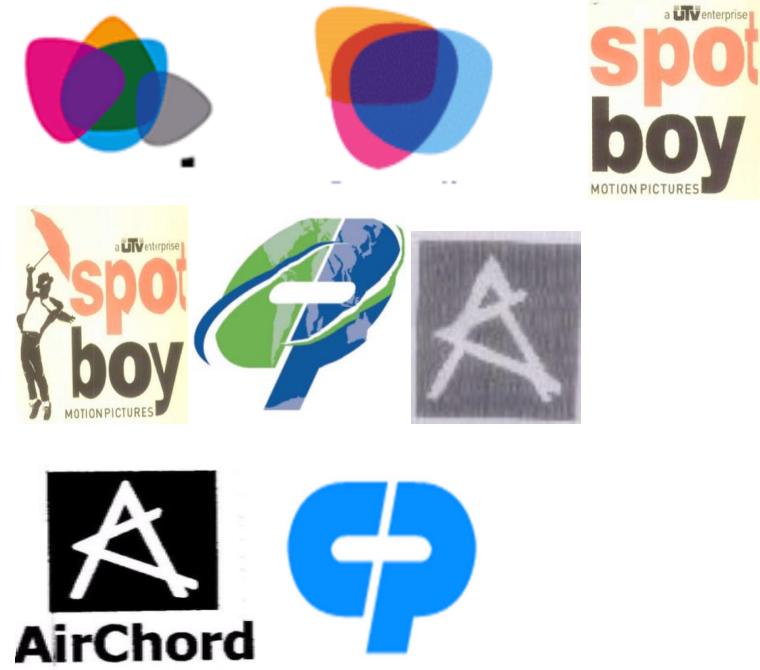

Figure 3 A typical set of trademark database images having some similarity

\subsection{GUI of The System}

The two snapshots of developed system are shown below:

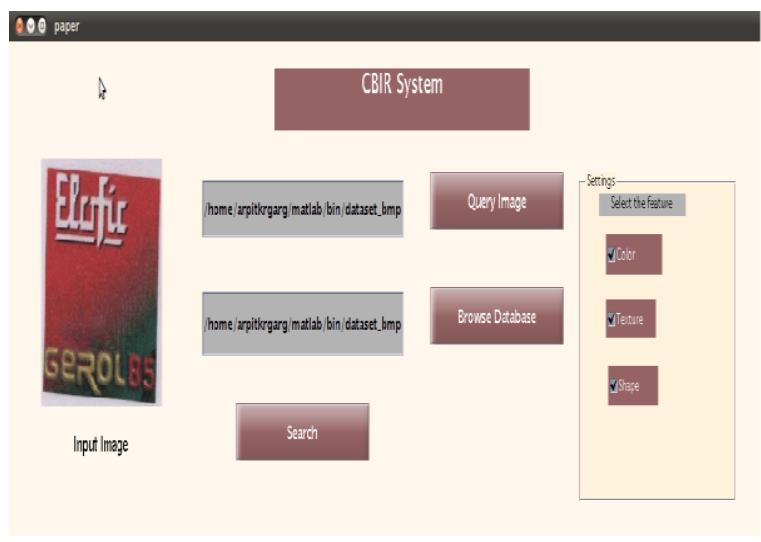

Figure 4 Snapshot 1 of the GUI of the proposed system

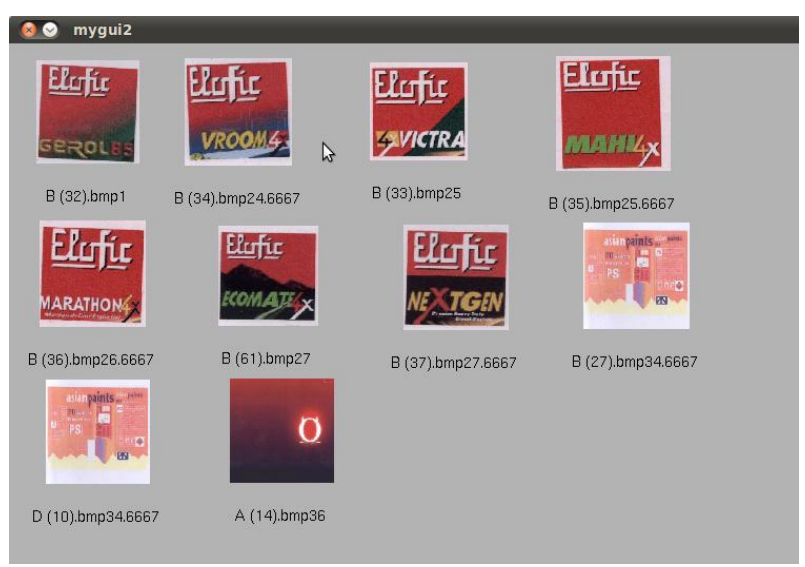

Figure 5 Snapshot 2 of the GUI of the proposed system

\section{CONCLUSION}

In this paper we have suggested a multi-featured combined approach of color, texture and shape information as attributes for trademark similarity matching which are combined by assigning pliable weights.

The approach shows the improvement in the results when weighted adjustment of features is used than when individual features are used independently. Clustering aids calculating recall and precision values and moderates the search space. Results arrived at are superior to the methods reported so far in the literature. 


\section{REFERENCES}

[1] Jan Schietse, John P. Eakins, Remco C. Veltkamp "Practice and Challenges in Trademark Image Retrieval", ACM ,2007

[2] Nidhi Singhal, Prof. Shishir K. Shandilya, "A Survey on: Content Based Image Retrieval Systems", International Journal of Computer Applications(09758887) Volume 4- No.2 July 2010

[3] T. Kato, "Database architecture for content-based image retrieval", Proceedings of SPIE Image Storage and Retrieval Systems, vol. 1662, pp. 112-123, 1992.

[4] J.K. Wu, C.P. Lam, B.M. Mehtre, Y.J. Gao, and A. Narasimhalu, "Content-based retrieval for trademark registration", Multimedia Tools Application, vol. 3, no. 3, pp. 245-267, 1996.

[5] J. P. Eakins, M.E. Graham, and J.M. Boardman, "Evaluation of a trademark image retrieval system", Information Retrieval Research, the 19th Annual BCS-IRSG Colloquium on IR Research, 1997

[6] M. Flickner et al "Query by image and video content" IEEE Computer 28(9), 23-32, Sep 1995

[7] S. Nandagopalan, Dr. B. S. Adiga, and N. Deepak "A Universal Model for Content-Based Image Retrieval" World Academy of Science, Engineering and Technology 462008

[8] Mr. Kondekar V. H., Mr. Kolkure V. S., Prof.Kore S.N "Image Retrieval Techniques based on Image
Features: A State of Art approach for CBIR", (IJCSIS) International Journal of Computer Science and Information Security, Vol. 7, No. 1, 2010.

[9] Rajshree S. Dubey, Rajnish Choubey, Joy Bhattacharjee, "Multi Feature Content Based Image Retrieval" International Journal on Computer Science and Engineering Vol. 02, No. 06, 2010, 2145-2149.

[10] Ranjeet Kumar, R.C.Tripathi, M.D. Tiwari “ A Comprehensive Study on Content Based Trademark Retrieval System", International Journal of Computer Applications (0975 - 8887)Volume 13- No.6, January 2011

[11] P.S.Suhasini, Dr. K.Sri Rama Krishna, Dr. I. V. Murali Krishna, "CBIR Using Color Histogram Processsing" Journal of Theoretical and Applied Information Technology, 2009

[12] N. S. Vassilieva "Content-based Image Retrieval Methods", ISSN 0361-7688, Programming and Computer Software, 2009.

[13] Tianhorng Chang and C.C Jay Kuo, Senior member IEEE, "Texture analysis and classification with treestructured wavelet transform" IEEE transactions on image processing, Volume 2, No. 4, October 1993.

[14] W.-Y. Kim and Y.-S. Kim, "A region based shape descriptor using Zernike moments". Signal Processing: Image Communication, Vol. 16, no. 1-2, 2000 pp. $95-102$. 